\title{
Difficultés des apprenants iraniens du FLE dans la gestion des finales verbales en/E/
}

\author{
Katayoon Katoozian \\ Université de la Sorbonne Nouvelle - Paris 3
}

\section{Introduction}

L'orthographe du français comme tout système d'écriture linguistique est mixte, composée de deux principes complémentaires : la phonographie qui assure la correspondance entre les unités graphiques et les unités non significatives de la langue (ex. phonèmes ou syllabes) et la sémiographie qui est responsable de la relation entre les unités graphiques et les unités de la langue porteuses de sens (ex. morphèmes, lexèmes et mots). La difficulté de l'orthographe française, classée parmi les plus compliquées du monde, est la conséquence du fonctionnement de ces deux principes : le premier par l'existence d'une polyvalence graphique de haut degré (un phonème peut être transcrit par plusieurs graphèmes et vice versa), le second par le recours aux divers procédés, dont l'homophonie (Katoozian, Analyse 59).

Ce que l'on appelle par tradition l'« orthographe grammaticale » est régie par des relations morphosyntaxiques qui sont pour la plupart silencieuses. L'homophonie des formes verbales en $/ \mathrm{E} /{ }^{2}$ en est l'exemple emblématique : ce sont les verbes du $1^{\text {er }}$ groupe, la conjugaison la plus « vivante » du français (Brissaud et Sandon 41), dont les formes de l'infinitif (manger), du participe passé (mangé, mangée, mangés, mangées), de l'imparfait (mangeais, mangeait, mangeaient), du passé simple (mangeai) et de la $2^{\mathrm{e}}$ personne $\mathrm{du}$ pluriel de l'indicatif présent (mangez) sonnent identiquement (homophones hétérographes). Les deux dernières formes mises à part par commodité, nous montrerons dans cet article ces trois oppositions fondamentales respectivement par des morphonogrammes de mode-temps correspondants : - ER, - É et - AI. Ces finales verbales homophones montrent d'une part l'opposition temporelle (passé composé vs imparfait) et d'autre part, l'opposition modale (infinitif vs participe passé). L'importance de ces oppositions est attribuable à leurs rôles dans la compréhension du français écrit et à leurs fréquences d'emploi dans la vie courante pour tout locuteur francophone/francographe, apprenants

1 Nommée également "orthographe de règle», "orthographe d'accord», " orthographe de principe», "orthographe relative», "orthographe logique», « orthographe syntaxique (Katoozian, Analyse 57).

${ }^{2}$ La distinction entre les timbres fermés $(/ \mathrm{e}, \varnothing, \mathrm{o} /)$ et ouverts $(/ \varepsilon, \propto, \diamond /)$ des voyelles dites « intermédiaires » du français en position accentuée se fait de plus en plus rare chez les Français et la réalisation de ces phonèmes est montrée à l'aide de l'archiphonème correspondant (/E, $\mathrm{E}, \mathrm{O} /)$. 
débutants ou experts confirmés (Meleuc et Fauchart 4). À ce propos Luzzati (Le français 56) donne l'exemple des phrases "l'enfant aimé sautait dans l'eau» et "l'enfant aimait sauter dans l'eau ». Cet auteur ajoute que la maîtrise de l'écriture et celle du sens vont de pair et que l'orthographe révèle la compréhension. Ainsi pour les exemples mentionnés, la phrase erronée $^{3}$ "*les enfants aimé sautait dans l'eau» est moins dramatique que la phrase "*les enfants aimer sauté dans l'eau » puisque la première est un simple problème orthographique tandis que la seconde est une violation du sens.

Ce «triangle infernal» (Brissaud, L'infinitif) constitue donc une difficulté orthographique majeure dont l'étude n'a pas manqué d'intérêt en FLM (Français Langue Maternelle). À la suite de deux études parallèles et transversales en 1996 auprès de 1269 élèves français de l'enseignement élémentaire et secondaire ${ }^{4}$ (observation d'un corpus d'erreurs relevées dans 180 rédactions et d'un test composé de 48 phrases lacunaires), les chercheurs ont remarqué que le secteur des finales verbales en/E/recueille, à la fin du secondaire, une erreur sur quatre qui consiste à utiliser une forme d'infinitif (—ER), de participe passé (—E) ou d'imparfait (— AI) à la place de la forme attendue (Brissaud, L'imparfait, L'infinitif, Travailler morphologie; Brissaud et Sandon). Cependant, une diminution très significative du nombre d'erreurs entre ces niveaux a été rapportée; ce qui est preuve de l'amélioration continue des performances au fil de la scolarité au secondaire. Quant à la compétition entre les formes en - ER, - É et - AI, c'était la forme variable en - É qui était privilégiée par l'échantillon de ces études; ce qui montre la propension des élèves à marquer cette forme en genre et en nombre du sujet. Une autre enquête identique menée auprès de 621 élèves français et canadiens de l'enseignement élémentaire et secondaire $^{5}$ (318 en France et 303 au Québec) a donné des résultats semblables (Chevrot, Brissaud, et Lefrançois; Brissaud, Chevrot, et Lefrançois). Mais ce n'est pas toujours la forme en - É qui est sélectionnée. D'autres chercheurs dans une étude auprès de 87 lycéens français de classes de $1^{\text {res }}$ scientifiques et littéraires ont noté des résultats différents : une performance significativement meilleure pour les structures induisant un infinitif que celles induisant un participe passé (Fayol et Pacton). Cette variété de résultats peut être due à la différence de méthodologie et de niveau des élèves de ces enquêtes.

Les études menées sur la compétence orthographique des apprenants du FLE (Français Langue Étrangère) montrent que les erreurs d'orthographe grammaticale sont parmi les plus fréquentes à cause des difficultés sui generis de ce secteur de l'orthographe du français (Katoozian,

\footnotetext{
${ }^{3}$ Les graphies erronées seront désormais précédées d'astérisque.

${ }^{4} \mathrm{Du} \mathrm{CM} 2$ (cours moyen deuxième année) à la $3 \mathrm{e}$.

${ }^{5}$ En France du CE2 (cours élémentaire deuxième année) à la 4e, au Québec de la $3^{\mathrm{e}}$ année du primaire au $2^{\mathrm{e}}$ secondaire.
} 
Analyse, Comparaison). Cependant, il manque d'études en FLE portant sur les difficultés des apprenants dans la gestion de l'homophonie des finales verbales en/E/. C'est pour cette raison que nous nous sommes penchée sur la question en poursuivant l'objectif d'étudier les compétences des apprenants iraniens du FLE dans ce secteur. Les deux premières questions de recherche qui émergent à la suite de cette revue d'articles sont les suivantes: dans un premier temps, nous voulons savoir comment se fait l'évolution de la compétence de gestion des finales verbales en/E/ : Est-ce que les apprenants iraniens progressent dans ce secteur de l'orthographe grammaticale du français? Ensuite, nous nous intéressons à connaître, parmi les formes en — ER, - É, et - AI, la forme la mieux réussie, autrement dit, la tendance des apprenants dans le choix de ces morphonogrammes de mode-temps.

L'accord avec le sujet de la phrase à travers le nombre important de formes erronées au participe passé marquées par le genre ou le nombre du sujet a également été rapporté par les recherches antérieures en FLM (Brissaud, L'imparfait, Réalisation de l'accord, L'infinitif; Brissaud et Sandon; Chevrot, Brissaud, et Lefrançois; Brissaud, Chevrot, et Lefrançois; Brissaud et Cogis). Également, la question de l'accord en personne a attiré l'attention des chercheurs pour savoir dans quelle mesure son acquisition est contrainte par les structures morphosyntaxiques et on a retrouvé pour l'imparfait un phénomène d'accord par proximité (*La grand-mère les regardaient) (Brissaud, Chevrot, et Lefrançois). Ces données nous ont amenées à poser la dernière question de cette recherche en deux parties : nous voulons savoir, premièrement, si dans les formes erronées en - É les apprenants de notre échantillon font l'accord avec le genre et le nombre du sujet de la phrase et deuxièmement, s'ils ont des problèmes d'accord en personne dans les formes en - AI.

L'objectif global de cette recherche consiste donc à «décrire»les difficultés des apprenants iraniens du FLE dans la gestion des finales verbales en/E/. Comme le précise Jaffré (17), «le choix méthodologique est dicté par un certain nombre de facteurs parmi lesquels on doit citer en tout premier lieu les objectifs de la recherche». Dans une recherche descriptive, comme son nom l'indique, il s'agit d'observer la situation didactique afin de la comprendre. Nous ne cherchons donc pas à trouver des rapports de causalité (relations cause-effet) comme c'est le cas dans les recherches expérimentales qui essayent de faire varier un ou plusieurs facteurs de la situation pour en étudier les effets (Hafeznia 64; Mialaret 98). Puisque nous n'avons pas eu la possibilité de suivre dans le temps les apprenants de notre échantillon, comme c'est le cas pour les recherches longitudinales, nous avons mené notre étude transversale dans une coupe de temps précise (automne 2015). 


\section{Méthodologie}

\subsection{Présentation du test}

L'outil d'observation de cette recherche était un test composé de 30 phrases lacunaires : 10 phrases pour chacune des cibles en - ER, — É, et - AI. Étant donné l'importance du cadre syntaxique dans l'émergence des erreurs, affirmée par les recherches antérieures en FLM (Jaffré et Bessonnat, Accord; Brissaud, Réalisation de l'accord), la structure de ces phrases était simple de type sujet+verbe suivi soit d'un groupe nominal au masculin singulier soit d'un adverbe. Les 10 phrases à l'infinitif étaient de régime direct (5 phrases de type aller+infinitif et 5 autres de type devoir+infinitif), les 10 phrases au participe passé étaient avec auxiliaire avoir employé sans accord et sans écran ${ }^{6}$ et parmi les 10 phrases à l'imparfait, 5 étaient de type nom+verbe+adjectif/adverbe et 5 autres de type nom +pronom +verbe où le nom et le pronom étaient de nombre et de genre différents pour tester si ce conflit d'accords empêche ou pas l'accord correct avec le sujet de la phrase. Quant au sujet de ces phrases et afin de répondre à la dernière question de cette recherche, nous avons fait nôtres les principes méthodologiques choisis par Chevrot, Brissaud, et Lefrançois en adoptant quatre types de sujet: masculin singulier (Jean, 9 phrases), féminin singulier (Marie, 9 phrases), masculin pluriel (Les garçons, 6 phrases) et féminin pluriel (Les filles, 6 phrases). L'ordre de ces phrases était aléatoire afin de ne pas influer sur le choix de telle ou telle autre cible.

Quant aux verbes, il s'agissait de dix verbes du $1^{\text {er }}$ groupe employé chacun à l'infinitif, au participe passé et à l'imparfait. Dans les recherches antérieures, un effet significatif de l'orientation statistique modale des verbes sélectionnés sur le choix entre les formes en - ER et - É a été rapporté (Brissaud, Chevrot, et Lefrançois; Fayol et Pacton). Dans le but d'éviter ce biais, tout d'abord, la fréquence des dix verbes choisis a été contrôlée : tous ces verbes ${ }^{7}$ se trouvent dans les Listes Orthographiques de Base $\mathrm{du}$ français (LOB) (Catach et Jejcic). Ces listes comprennent 4000 formes des 1620 mots qui représentent plus de $90 \%$ des potentialités d'occurrence dans les textes français (ibid. 4). L'avantage des LOB s'explique par le fait qu'elles séparent les mots vedettes des formes fléchies avec indication de la nature, du genre et du nombre des mots (Katoozian, Analyse 233). En outre, six de ces verbes ${ }^{8}$ se trouvent parmi les 100 verbes les plus fréquents du français écrit (recensés par le Trésor de la Langue Française) et oral (recensés par l'Élaboration du Français Fondamental) (Meleuc et Fauchart 7072). Parmi les bases de données informatisées, nous avons choisi la plus

\footnotetext{
${ }^{6}$ Trois types sont possibles : 1- sans accord et sans écran (Il a mangé une pomme) ; 2 sans accord avec écran (Il leur a demandé un stylo) ; 3- avec accord (Il les a cherchés).

7 Trouver, donner, aimer, demander, appeler, changer, considérer, traiter, élever, dépenser.

${ }^{8}$ Trouver, donner, aimer, demander, appeler, changer.
} 
récente, Lexique3 (New et al.) dont nous avons consulté la dernière version sur internet (version 3.80) ${ }^{9}$. Tous les verbes sélectionnés se trouvent aussi dans cette base qui contient les formes fléchies avec estimations de fréquence plus actuelles basées sur deux grands ensembles de corpus: textes littéraires et dialogues de films et de séries.

À l'étape suivante, l'orientation modale des verbes choisis (infinitif vs participe passé $)^{10}$ a été contrôlée de sorte que la fréquence d'occurrence de chacun d'eux à l'infinitif et au participe passé (toutes les formes verbales en - é, - éé, - és, - ées) soit approximativement équivalente. Cette étape, comme la précédente, a été réalisée parallèlement avec les LOB et la base de données Lexique3.

\subsection{Présentation de l'échantillon}

Notre échantillon était composé de 113 apprenants iraniens adultes (hommes et femmes) du FLE inscrits à quatre instituts de langue de Téhéran ${ }^{11}$ répartis comme suit : 38 apprenants de niveau A1 acquis, 43 de niveau A2 acquis et 32 de niveau B1 acquis. Tous ces apprenants avaient étudié les manuels "Le Nonvean Taxi!»" et «Le Nonvel Édito ${ }^{13}$ correspondants à leur niveau respectif. Ce sont des méthodes récentes de l'enseignement $\mathrm{du}$ français qui, s'adressant à un public de grands adolescents et d'adultes, s'inscrivent dans la perspective actionnelle et proposent une grammaire plus explicite (à travers des tableaux de grammaire et des exercices de systématisation linguistique). L'enseignement des temps verbaux (passé composé et imparfait) commence dès le premier volume (Le Nouveau Taxi! 1) et dans les volumes suivants les apprenants révisent et approfondissent leurs connaissances de ces temps verbaux. Le dernier volume de ces manuels couvre environ 120 heures d'enseignements et s'adresse aux apprenants de niveau B1 ayant suivi 200 heures

\footnotetext{
${ }^{9}$ http:/ / www.lexique.org/

${ }^{10} \mathrm{La}$ fréquence du morphonogramme -AI à l'intérieur des verbes du 1 er groupe étant moins importante par rapport à celle de l'opposition modale « infinitif vs participe passé » (Brissaud et Sandon 41).

${ }_{11}$ Peu nombreux étaient les instituts qui, lors de cette phase difficile de collecte de données, nous ont prêté leur collaboration. Nous tenons à les remercier sincèrement.

12 Pour le niveau A1 acquis : CAPELLE G., MENAND R. (2009), Le Nonvean Taxi! 1, Paris, Hachette (Coll. Français Langue Étrangère) ; pour le niveau A2 acquis : MENAND R. et al. (2009) Le Nowvean Taxi! 2, Paris, Hachette (Coll. Français Langue Étrangère) ; pour le niveau B1 acquis : MENAND R. (2010) Le Nouveau Taxi! 3, Paris, Hachette (Coll. Français Langue Étrangère).

13 Pour le niveau B1 acquis: HEU É. et al. (2012), Le Nonvel Édito. Nivean B1, Paris, Didier.
} 
d'apprentissage du français. Il est à préciser que le manuel «Le Nouvel Édito » vise seulement les niveaux B1 et B2 du CERCL ${ }^{14}$.

Le test de la recherche a été dicté à ces apprenants en répétant deux fois chaque phrase. Les copies ainsi ramassées ont été codifiées et corrigées en attribuant 1 point à chaque réponse correcte et 0 point aux réponses incorrectes. Vu le nombre total des phrases du test, la note globale a été comptée à partir de 30. Les erreurs d'accentuation et d'orthographe lexicale ont été tolérées. Seules les erreurs de type phonétique (*appoller pour appeler) et les erreurs de segmentation (*est lever pour élever) donnaient la permission d'enlever de point et ceci malgré la finale verbale correcte parfois ${ }^{15}$. En effet, ces types d'erreur sont classés dans la quasi-totalité des systèmes de classification d'erreurs d'orthographe française comme "préorthographiques » : ce sont des erreurs de langue qui ne dépendent pas de l'orthographe d'où leur importance et aussi leur fréquence élevée chez les apprenants du FLE (Katoozian, Analyse, Comparaison, Une revue d'articles, Revue critique).

\section{Résultats}

\subsection{Finales verbales en/E/ : compétence stagnante}

Le tableau 1 fournit les éléments de réponse à la première question de cette recherche («Comment se fait l'évolution de la compétence de gestion des finales verbales en/E/?») en comparant les notes obtenues par les apprenants de chaque niveau pour chaque cible et pour le test entier. Force est de constater que ces notes ont été accordées aux réponses correctes pour les cibles attendues et ne comprennent pas les erreurs d'accord en genre, en nombre et en personne. Étant donné le nombre de phrases de chaque cible, sa note est comptée sur 10 et vu le nombre total des cibles, la note globale s'arrête à 30 (les chiffres entre parenthèses dans le rang des niveaux indiquent l'effectif des apprenants).

\footnotetext{
${ }^{14}$ Cadre européen commun de référence pour les langues

15 Afin de mieux connaitre les types d'erreurs d'orthographe du français voir Katoozian (Revue critique).
} 


\begin{tabular}{|c|c|c|c|}
\hline Niveaux & $\begin{array}{c}\mathrm{A} 1 \\
(\mathrm{n}=38)\end{array}$ & $\begin{array}{c}\mathrm{A} 2 \\
(\mathrm{n}=43)\end{array}$ & $\begin{array}{c}\text { B1 } \\
(\mathrm{n}=32)\end{array}$ \\
\hline - ER & 7.9 & 8.4 & 8.7 \\
\hline SE & 0.4 & 0.3 & 0.3 \\
\hline - ${ }^{\prime} \quad \bar{x}$ & 7.6 & 7.9 & 8.3 \\
\hline SE & 0.5 & 0.4 & 0.4 \\
\hline $\begin{array}{ll} & \bar{x} \\
-\mathrm{AI} & \end{array}$ & 5.8 & 6.7 & 6.4 \\
\hline SE & 0.7 & 0.6 & 0.7 \\
\hline $\begin{array}{lr} & \bar{x} \\
\text { Total } & \end{array}$ & $\begin{array}{l}21.3 \\
1.3\end{array}$ & $\begin{array}{l}23.1 \\
1.1\end{array}$ & 23.4 \\
\hline
\end{tabular}

Tableau 1 : Description statistique des notes (cible par niveau)

Tout d'abord, l'étude des erreurs types, étant donné leur faiblesse, montre que ces moyennes possèdent une valeur suffisante. Une première lecture de ce tableau permet de constater une amélioration des moyennes pour chaque cible et pour le test entier (sauf pour la cible - AI où la situation est apparemment meilleure chez les apprenants de A2). Pour savoir si ces améliorations sont significatives nous avons conduit, à l'aide du logiciel statistique SPSS, des analyses de variance (One-way ANOVA) dont le résultat montre que ces différences ne sont pas significatives ni pour la note globale ni pour aucune de ces cibles, donc aucun effet significatif de niveau ne s'observe sur l'amélioration de la compétence de gestion des finales verbales en/E/chez les apprenants de notre échantillon (cible $\mathrm{ER}: \mathrm{F}[2,110]=1.308, \mathrm{p}>0.275$; cible $-\mathrm{E}: \mathrm{F}[2,110]=0.824, \mathrm{p}>0.441$; cible - AI : F $[2,110]=0.513, \mathrm{p}>0.600$; note globale $: \mathrm{F}[2,110]=0.909$, $\mathrm{p}>0.406)$.

\subsection{Tendance pour l'invariabilité}

\subsubsection{De la forme en - ER privilégiée...}

La lecture verticale du tableau 1 révèle que pour chaque niveau la forme en - ER est mieux réussie suivie respectivement des formes en - É et en - AI. Mais ce ne sont pas les seules formes produites par les apprenants : le tableau 2 tente de répondre à la deuxième question de notre recherche («Parmi les formes en — ER, — É, et — AI laquelle est mieux réussie? ») tout en présentant les autres types d'erreurs avec leur fréquence de distribution (les chiffres en gras correspondent aux taux de réussite). 


\begin{tabular}{|c|c|c|c|c|c|c|c|c|c|}
\hline \multirow{2}{*}{$\begin{array}{l}\text { Cibles/Niveaux } \\
\text { Types } \\
\text { d'erreurs }\end{array}$} & \multicolumn{3}{|c|}{ - ER $(\%)$} & \multicolumn{3}{|c|}{ 一É $(\%)$} & \multicolumn{3}{|c|}{ - AI $(\%)$} \\
\hline & A1 & A2 & B1 & A1 & A2 & B1 & A1 & A2 & B1 \\
\hline$-\mathrm{ER}$ & 79.0 & 84.4 & 87.2 & 4.7 & 3.3 & 3.4 & 7.9 & 1.9 & 5.6 \\
\hline 一É & 3.4 & 4.0 & 3.4 & 75.5 & 79.3 & 83.1 & 3.2 & 2.8 & 7.5 \\
\hline$-\mathrm{AI}$ & 1.6 & 1.9 & 1.6 & 1.1 & 3.7 & 1.6 & 58.4 & 67.0 & 64.1 \\
\hline$-\mathrm{e}$ & 2.9 & 1.2 & 3.1 & 4.0 & 3.7 & 3.1 & 15.8 & 16.3 & 19.4 \\
\hline Autres (- ez) & - & - & - & - & - & - & - & 0.7 & - \\
\hline $\begin{array}{c}\text { Erreurs } \\
\text { phonétiques }\end{array}$ & 5.8 & 5.1 & 2.5 & 7.1 & 6.5 & 4.4 & 7.4 & 6.1 & 2.2 \\
\hline $\begin{array}{c}\text { Erreurs de } \\
\text { segmentation }\end{array}$ & 3.4 & 2.1 & 0.6 & 3.4 & 2.1 & 2.5 & 3.4 & 3.5 & 0.3 \\
\hline Sans-réponses & 4.0 & 1.4 & 1.6 & 4.2 & 1.4 & 1.9 & 4.0 & 1.9 & 1.0 \\
\hline
\end{tabular}

Tableau 2 : Distribution des types d'erreurs par cible et niveau

La forme neutre en -e, les erreurs phonétiques et les erreurs de segmentation sont présentes à côté des erreurs de cibles sans compter les sans-réponses et une forme marginale (-ez) une seule fois observée chez les apprenants A2 et pour la cible -AI. Le taux de réussite le plus élevé concerne la cible -ER pour le niveau B1 $(87.2 \%)$ et le moins élevé concerne la cible -AI pour le niveau A1 (58.4\%), d'ailleurs confirmés par le tableau 1. Pour la cible -ER, si ce sont les erreurs phonétiques qui sont les plus fréquentes pour les niveaux A1 et A2 (respectivement $5.8 \%$ et $5.1 \%$ ), les formes en -É occupent le premier rang des erreurs chez le niveau B1 $(3.4 \%)$ et comptent également parmi les plus fréquentes chez les deux autres niveaux $(3.4 \%$ pour $\mathrm{A} 1,4.0 \%$ pour $\mathrm{A} 2)$.

Quant à la cible -É, l'erreur phonétique est encore la plus fréquente pour tous les niveaux (7.1\% pour A1, $6.5 \%$ pour A2, $4.4 \%$ pour B1). La forme en -ER constitue la tendance dominante pour les niveaux A1 (4.7\%) et B1 (3.4\%). Pour le niveau A2 et après les erreurs phonétiques, ce sont les formes en -AI et $-e$ qui sont les plus fréquentes ( $3.7 \%$ chacune). En ce qui concerne la cible -AI, c'est la forme neutre en -e qui est désormais la forme la plus choisie avec une fréquence nettement supérieure $(15.8 \%$ pour A1, 16.3 \% pour A2, $19.4 \%$ pour B1). Après cette forme, l'erreur la plus fréquente pour le niveau A1 est la forme en -ER (7.9\%), pour le niveau A2 l'erreur phonétique $(6.1 \%)$ et pour le niveau B1 la forme en -É 
(7.5\%). Des trois formes en -ER, -É et -AI, cette dernière est parmi les moins sélectionnées quand la cible est -ER ou -É (sauf pour le niveau A2 quand la cible est -É).

\subsection{2. ... À l'imperméabilité des formes en -É aux marques de genre et de nombre du sujet}

Le tableau 3 permet de répondre à la dernière question de notre recherche ( Dans les formes en -É, est-ce que les apprenants font l'accord avec le genre et le nombre sujet ? Est-ce qu'ils ont des problèmes d'accord en personne dans les formes en -AI?»). Les chiffres en gras correspondent aux taux de réussite. 


\begin{tabular}{|c|c|c|c|c|c|c|c|c|c|c|}
\hline \multirow{2}{*}{\multicolumn{2}{|c|}{$\begin{array}{l}\text { Cibles/Niveaux } \\
\text { Types } \\
\text { d'erreurs }\end{array}$}} & \multicolumn{3}{|c|}{-ER (\%) } & \multicolumn{3}{|c|}{-É (\%) } & \multicolumn{3}{|c|}{-AI $(\%)$} \\
\hline & & A1 & A2 & B1 & A1 & A 2 & B1 & A1 & A2 & B1 \\
\hline & -ER & $\begin{array}{c}79 . \\
0\end{array}$ & $\begin{array}{c}84 . \\
4\end{array}$ & $\begin{array}{c}87 . \\
2\end{array}$ & 4.7 & 3.3 & 3.4 & 7.9 & 1.9 & 5.6 \\
\hline \multirow{6}{*}{-É } & $\begin{array}{l}\text {-é/-ée,-és,- } \\
\text { ées sans } \\
\text { rapport } \\
\text { avec le } \\
\text { sujet }\end{array}$ & 2.9 & 3.5 & 2.8 & - & - & - & 2.6 & 2.3 & 4.7 \\
\hline & -é & - & - & - & $\begin{array}{c}69 . \\
5\end{array}$ & $\begin{array}{c}73 . \\
3\end{array}$ & $\begin{array}{c}75 . \\
6 \\
\end{array}$ & - & - & - \\
\hline & $\begin{array}{l}\text {-ée,-és,-ées } \\
\text { sans } \\
\text { rapport } \\
\text { avec le } \\
\text { sujet }\end{array}$ & - & - & - & 1.8 & 1.6 & 2.8 & - & - & - \\
\hline & -ée (Marie) & 0.5 & 0.5 & 0.3 & 2.1 & 0.9 & 2.8 & 0.3 & - & 1.3 \\
\hline & $\begin{array}{l}\text {-és (Les } \\
\text { garçons) }\end{array}$ & - & - & - & 1.1 & 3.0 & 0.3 & 0.3 & - & 0.9 \\
\hline & $\begin{array}{c}\text {-ées (Les } \\
\text { filles) }\end{array}$ & - & - & 0.3 & 1.1 & 0.5 & 1.6 & - & 0.5 & 0.6 \\
\hline \multirow{6}{*}{$\begin{array}{c}- \\
\text { A } \\
\text { I }\end{array}$} & $\begin{array}{l}\text { Accord } \\
\text { correct }\end{array}$ & 0.5 & 1.2 & 0.6 & 0.8 & 3.5 & 1.3 & $\begin{array}{c}50 . \\
5\end{array}$ & $\begin{array}{c}60 . \\
0\end{array}$ & $\begin{array}{c}55 . \\
0\end{array}$ \\
\hline & $\begin{array}{c}\text { Sujet } \\
\text { sg/verbe pl }\end{array}$ & - & - & 0.6 & - & - & - & - & - & - \\
\hline & $\begin{array}{c}\text { Sujet } \\
\mathrm{sg} / \text { verbe } \mathrm{pl} \\
\text { (écran) }\end{array}$ & - & - & - & - & - & - & 0.8 & 0.7 & 0.3 \\
\hline & $\begin{array}{c}\text { Sujet } \\
\mathrm{pl} / \text { verbe sg }\end{array}$ & 1.1 & 0.7 & 0.3 & 0.3 & 0.2 & 0.3 & 1.8 & 1.4 & 2.2 \\
\hline & $\begin{array}{c}\text { Sujet } \\
\mathrm{pl} / \text { verbe sg } \\
\text { (écran) }\end{array}$ & - & - & - & - & - & - & 1.6 & 2.8 & 2.5 \\
\hline & Autres & - & - & - & - & - & - & 3.7 & 2.1 & 4.1 \\
\hline & Autres & $\begin{array}{c}16 . \\
1\end{array}$ & 9.8 & 7.8 & $\begin{array}{c}18 . \\
7\end{array}$ & $\begin{array}{c}13 . \\
7\end{array}$ & $\begin{array}{c}11 . \\
9\end{array}$ & $\begin{array}{c}30 . \\
6\end{array}$ & $\begin{array}{c}28 . \\
5\end{array}$ & $\begin{array}{c}22 . \\
9\end{array}$ \\
\hline
\end{tabular}

Tableau 3 : Distribution des types d'erreurs d'accord par cible et niveau 
Ici nous avons reproduit le tableau 2 en apportant les modifications suivantes: 1- La fréquence des formes en -e et -ez ainsi que les erreurs phonétiques, les erreurs de segmentation et les sans-réponses une fois additionnées, nous les avons regroupées sous le label «Autres» au dernier rang du tableau. 2- Parmi les formes en -É pour les cibles -ER et -AI nous avons compté d'un côté les formes en -é ou les formes en -éé, -és, -ées sans rapport avec le sujet de la phrase et de l'autre, les formes en -éé, -és, -ées accordées avec le sujet de la phrase (respectivement Marie, Les garcons, Les filles). Pour la cible -É, puisque la forme attendue est $-e ́$ (les taux de réussite mis en gras dans le tableau), nous avons compté séparément les formes en éé, -és, -ées sans rapport avec le sujet de la phrase et les formes accordées.

Quant à la forme en -AI, par le label «Accord correct» nous voulons dire tous les rapports corrects de personne entre le sujet de la phrase et la forme verbale produite. Le label «Autres» au dernier rang des erreurs en $\mathrm{AI}$ indique d'une part les formes portant la marque de la $2^{\mathrm{e}}$ personne du singulier quand le sujet de la phrase est Jean ou Marie et d'autre part, l'absence de marque de personne. C'est pour ces modifications que les taux de réussite mis en gras pour les cibles -É et -AI dans ce tableau diffèrent de ceux indiqués dans le tableau 2.

Parmi les formes en -É, on constate que la fréquence de la forme non marquée $(-\hat{e})$ et des formes sans rapport avec le sujet de la phrase quand la cible est -ER ou -AI est supérieure à celle des formes portant les marques de genre et de nombre du sujet (cible -ER : $2.9 \%$ vs $0.5 \%$ pour A1, $3.5 \%$ vs $0.5 \%$ pour $\mathrm{A} 2,2.8 \%$ vs $0.6 \%$ pour $\mathrm{B} 1$; cible $-\mathrm{AI}: 2.6 \%$ vs $0.6 \%$ pour A1, $2.3 \%$ vs $0.5 \%$ pour A2, $4.7 \%$ vs $2.8 \%$ pour B1). La situation est identique quand la cible est -É $(71.3 \%$ vs $4.3 \%$ pour A1, $74.9 \%$ vs $4.4 \%$ pour $\mathrm{A} 2,78.4 \%$ vs $4.7 \%$ pour B1).

Pour ce qui est de l'accord en personne entre le sujet et le verbe, c'est la tendance pour l'accord correct qui est dominante (cible -ER: $0.5 \%$ vs $1.1 \%$ pour $\mathrm{A} 1,1.2 \%$ vs $0.7 \%$ pour $\mathrm{A} 2,1.2 \%$ vs $0.3 \%$ pour B1 ; cible -É : $0.8 \%$ vs $0.3 \%$ pour $\mathrm{A} 1,3.5 \%$ vs $0.2 \%$ pour $\mathrm{A} 2,1.3 \%$ vs $0.3 \%$ pour $\mathrm{B} 1$; cible -AI : $50.5 \%$ vs $7.9 \%$ pour A1, $60.0 \%$ vs $7.0 \%$ pour A2, $55.0 \%$ vs $9.1 \%$ pour B1).

\section{Discussion}

\subsection{Gestion de l'homophonie verbale en /E/ : spécificités du FLE}

De la comparaison entre les résultats obtenus de notre recherche ${ }^{16}$ et ceux rapportés par les recherches antérieures en $\mathrm{FLM}^{17}$, émergent quelques

\footnotetext{
16 Voir Section 3.

17 Voir Introduction.
} 
distinctions majeures, propres à notre échantillon d'apprenants non francophones: tendance pour l'invariabilité (fréquence élevée de la forme en -ER et des formes non marquées de -É), propension à produire une forme neutre $(-e)$, stagnation de la compétence de gestion de l'homophonie verbale en $/ \mathrm{E} /$ et persistance des erreurs préorthographiques.

Dans le trio «imparfait/participe passé/infinitif», c'était ce dernier, forme «prototypique » du verbe (Brissaud et Sandon 50), qui constituait le premier choix des apprenants de notre échantillon ${ }^{18}$. S'observant aussi chez les adultes francophones, David (224) nomme ce type d'erreur comme «erreur de l'expert». En effet, la cause de son apparition chez l'expert ne peut pas être la méconnaissance des règles, mais les conditions d'énonciations écrites et encore plus le «poids excessif des normes orthographiques» (ibid. 231). La propension des apprenants de FLE de notre échantillon pour l'infinitif pourrait ainsi s'expliquer que cette forme lemmatisée du verbe dans les dictionnaires est la première forme qu'ils rencontrent, chronologiquement parlant. Cette forme canonique du verbe qui constitue le lien entre lexique et grammaire devient le critère initial de repérage des verbes (Meleuc et Fauchart 60). C’est le problème engendré par la classification traditionnelle des verbes, fondée à la fois sur la nature de l'infinitif et les spécificités orthographiques de sa conjugaison. Ainsi, l'infinitif devient pour l'apprenant de FLE un indicateur univoque du groupe et du type de conjugaison qui constitue ce groupe (Surcouf 93, 103).

Cette tendance pour l'invariabilité va dans le même sens que l'absence d'accord avec le genre et le nombre du sujet de la phrase quand les apprenants optent pour la forme non marquée «-é». Là où les chercheurs parlent du «souci morphologique » de l'élève francophone dans le choix de la forme -é pour la marquer du genre et du nombre du sujet de la phrase (Chevrot, Brissaud, et Lefrançois 64-65), l'apprenant iranien, lui, préfère encore l'invariabilité. Ceci pourrait s'expliquer par l'absence du genre en tant que catégorie grammaticale en persan. Cependant, cette hypothèse devrait être vérifiée par des recherches semblables auprès $\mathbf{d}$ 'apprenants de langues maternelles différentes.

La forme en -e, ce morphème de personne ( $1^{\text {re }}$ et $3^{\text {e }}$ personne de l'indicatif présent des verbes en -er), constituait le choix par excellence des apprenants de notre échantillon quand la cible était -AI ${ }^{19}$. Malgré l'absence dans notre corpus d'erreurs de combinaisons incompatibles de marques de

\footnotetext{
$18 \mathrm{Au}$ moment de la passation du test de quelques-uns d'entre eux nous avons entendu dire : «Tout ça c'est de l'infinitif! Pourquoi donc continuer?!»

${ }^{19}$ Il pourrait s'agir de la forme -é non accentuée mais étant donné la coexistence de ces deux formes dans les copies des apprenants, nous avons classé et codifié la forme non accentuée à part.
} 
mode et de personne (*-ers, *-ert, *-éent) tout comme dans le corpus FLM (Brissaud et Sandon), la présence de la forme neutre en -e, non rapportée par les recherches antérieures en FLM, montre que chez l'apprenant qui l'a produite la connaissance phonie-graphie manque ou ne fonctionne pas bien (trouve / $t \mathrm{R} w /$ vs trouvait $/ t \mathrm{R} u v \mathrm{E} /$ ). À cette méconnaissance des règles phonographiques s'ajoutent la non-reconnaissance du mot et les problèmes d'ordre phonique, erreurs préorthographiques généralement fréquentes chez les apprenants non francophones (Katoozian, Analyse, Comparaison, Une revue d'articles).

\subsection{Et que disent les programmes?}

Dans les niveaux de références du CECRL pour le français, un chapitre est consacré à la «matière graphique » (Beacco et Porquier, A1 139-155 ; Beacco et Porquier, A2 171-185; Beacco et al. 225-255). Ce chapitre est composé de deux parties distinctes, compétence orthoépique et compétence orthographique. L'orthographe des formes verbales constitue une des composantes de la compétence orthographique qui traite les problèmes liés aux radicaux et aux désinences. L'ensemble du système de la morphologie verbale est présent dès $\mathrm{A} 1:$ «Ce qui change véritablement, c'est l'introduction des temps verbaux. A1 et A2 ne comportent que le présent qui autorise l'accès au passé (passé composé) comme au futur (futur périphrastique). Les bases de l'imparfait, du subjonctif présent et du futur simple apparaissent à compter de B1»(Luzzati, L'orthographe 15). Cependant l'identification de certaines formes et de certaines valeurs de l'imparfait ainsi que son utilisation pour certains verbes sont décrites comme compétence morphologique pour le niveau A2 (Beacco et Porquier, $A 2106$ sqq). En ce qui concerne l'orthographe et toujours pour le même niveau, l'identification ${ }^{20}$ de l'orthographe grammaticale de l'imparfait figure parmi les compétences à acquérir de l'orthographe grammaticale (ibid. 183 sqq). Force est de constater que cette même «identification » n'a pas fonctionné pour les apprenants A2 et B1 acquis de notre échantillon ${ }^{21}$. En FLM aussi et malgré la régularité morphologique de l'imparfait (Meleuc et Fauchart 135-139), son acquisition, posant problème, est considérée comme dépendante de celles de ses formes homophones les plus fréquemment utilisées (Brissaud, L’imparfait 63). En dépit de cette difficulté et contrairement aux élèves francophones (entre autres, Brissaud, Chevrot, et Lefrançois), les apprenants de notre échantillon réalisent

${ }^{20}$ Cependant, la leçon 17 de l'unité 5 du manuel Le Nowveau Taxi! 2 (p.55) et son cahier d'exercices (p.36), étudiés par les apprenants A2 acquis de notre échantillon, invitent les apprenants à employer l'imparfait. C'est le même cas et cela va de soi, pour le dossier 1 de l'unité 1 du manuel Le Nonvel Édito. Niveau B1 (pp.18-19) et son cahier d'exercices (pp.5-7), étudiés par les apprenants B1 acquis de notre échantillon.

${ }^{21}$ D'où leur question "comment distinguer s'il faut employer l'imparfait?" après la séance de passation du test. 
correctement l'accord de la personne du verbe à l'imparfait avec son sujet et la présence de l'écran ne leur a pas causé des difficultés.

\section{Conclusion}

La moyenne globale des notes des apprenants de notre échantillon, tout niveau compris, est supérieure à 20 (sur 30) et ce malgré la difficulté inhérente de ce secteur de l'homophonie des finales verbales en /E/. Cependant, comme c'est le cas pour tout système éducatif, on s'attend à une amélioration des compétences à travers ces niveaux d'apprentissage. Nous avons également observé chez ces apprenants un nombre considérable d'erreurs d'orthographe lexicale et d'erreurs d'accentuation que nous n'avons pas pu traiter, l'objectif de notre recherche ne nous l'ayant pas permis. La marginalisation de l'enseignement de l'orthographe dans les approches communicatives et la perspective actionnelle qui mettent l'accent avant tout sur le «sens » et la «communication orale » (Cuq et Gruca 264 sqq), devrait être remise en cause. La perspective actionnelle, dans laquelle s'inscrivent les deux manuels utilisés par les apprenants de notre échantillon ${ }^{22}$, " considère l'apprenant comme un acteur social ayant à accomplir des tâches à l'intérieur d'un domaine particulier » (ibid. 269) et ne laisse donc aucune place à l'enseignement-apprentissage approfondi de l'orthographe. Suleiman et Malkawi après l'examen d'une trentaine de méthodes FLE publiées en France entre 1990 et 2011, trouvent un nombre très limité d'activités orthographiques et tirent l'alarme sur le «niveau médiocre à l'écrit des étudiants jordaniens et, d'une manière générale, de la nouvelle génération de francophones » (ibid. 125). Luzzati (Le français 195) fait aussi mention de cette absence de l'enseignementapprentissage de l'orthographe en FLE: «L'objectif est d'abord d'apprendre à communiquer, et dans un second temps seulement d'apprendre à "graphier" ». Le taux élevé d'erreurs d'orthographe grammaticale ainsi que d'erreurs préorthographiques (erreurs de segmentation et erreurs phonétiques) rapporté par les recherches antérieures en FLE (Katoozian, Analyse, Comparaison, Une revue d'articles), nous amène à redéfinir la place de l'orthographe dans la méthodologie de l'enseignement-apprentissage du FLE en général et dans les méthodes actuelles du français en particulier.

Une telle redéfinition pourrait aller dans le sens de favoriser la " démarche réflexive » qui invite les apprenants à réfléchir sur leur façon d'écrire (Katoozian, Analyse 341-345). Dans cette démarche et pour enseigner le principe phonographique de l'orthographe française, on invite les apprenants à «découvrir » eux-mêmes les différents graphèmes possibles pour transcrire un tel phonème et à découvrir la distribution et la position de chaque graphème ainsi trouvé. Une telle procédure les

22 Voir notes 12 et 13 . 
empêcherait, par exemple, de produire la forme neutre -e pour le phonème /E/. En ce qui concerne le principe sémiographique de l'orthographe française aussi cette démarche heuristique qui passe par la résolution de problèmes, l'observation et la réflexion, pourrait largement contribuer à améliorer la compétence orthographique des apprenants (Haas et Lorrot). Brissaud et al. (237-259) donnent un exemple de ce type de séances pour le FLM qui invitent les élèves, dans le secteur de l'homophonie verbale en /E/ et afin de distinguer l'infinitif de l'imparfait, à remplacer les verbes en /E/ (aimer) par les verbes en /R/ (vendre). L'efficacité de cette procédure « analogique » a été également démontrée par David, Guyon, et Brissaud dans une étude auprès de 195 élèves français du primaire (CM2).

À côté de cette démarche réflexive certains auteurs préconisent en outre la «traduction», méthode utilisée autrefois dans l'enseignement des langues anciennes. L'étude d'une séquence telle je sais qu'il l'a vu(e) passe soit par une décomposition grammaticale soit par une traduction : "C'est une condition nécessaire pour traiter aussi bien des marques de genre et de nombre que la question particulièrement délicate en français des homophones grammaticaux » (Luzzati, Le français 59). Ainsi, comme le proposent Meleuc et Fauchart (92), il faut amener les apprenants à découvrir que l'emploi de l'infinitif ou du participe passé entraîne un «sens différent » de la phrase.

Ces activités d'éveil au langage et de traduction peuvent être menées à travers des «ateliers de négociation graphique » (Haas et Lorrot) à partir des erreurs produites. Ces ateliers constituent de véritables observatoires de l'apprentissage du raisonnement qui invitent les apprenants à «analyser» la langue. C'est dans le cadre de ces ateliers que les enseignants trouvent l'occasion de prêter l'oreille aux représentations des apprenants sur les formes produites à travers des «entretiens métagraphiques» menés avec eux. Les instituts de langue consultés dans cette recherche ne nous ont pas donné l'autorisation de profiter davantage du temps de leurs classes afin de savoir comment les apprenants commentent les formes produites. Il serait souhaitable que les recherches ultérieures se penchent sur cette question délicate. 


\section{Bibliographie}

Beacco, Jean-Claude, et Rémy Porquier. Niveau A2 pour le français, un référentiel. Paris : Didier, 2008.

. Niveau A1 pour le français, un référentiel. Paris : Didier, 2007.

Beacco, Jean-Claude, Béatrice Blin, Emmanuelle Houles, Sylvie Lepage, et Patrick Riba. Niveau B1 pour le français, un référentiel. Paris : Didier, 2011.

Brissaud, Catherine. "Travailler la morphologie écrite du verbe au collège ». Le français aujourd'bui 139 (2002) : 59-66.

. "L'infinitif en -er : difficultés orthographiques au collège ». La Linguistique 35-2 (1999) : 61-78.

"La réalisation de l'accord du participe passé employé avec avoir. De l'influence de quelques variables linguistiques et sociales ». Langage \& société 88 (1999) : 5-24.

. "L'imparfait et ses concurrents, du CM2 à la troisième ». Le français aujourd'bui 122 (1998) : 62-70.

Brissaud, Catherine, Danièle Cogis, Jean-Pierre Jaffré, Jean-Christophe Pellat, et Michel Fayol. Comment enseigner l'orthographe aujourd'bui. Paris : Hatier, 2011.

Brissaud, Catherine, et Danièle Cogis. «L'accord du participe passé. Reconsidération d'unproblème ancien à la lumière de données récentes sur l'acquisition ». Actes du CMLF $2008-1^{\text {er }}$ Congrès Mondial de Linguistique Française (2008): 413-424. Web [DOI $10.1051 / \mathrm{cmlf08105]}$.

Brissaud, Catherine, Jean-Pierre Chevrot, et Pascale Lefrançois. «Les formes verbales homophones en /E/ entre 8 et 15 ans: contraintes et conflits dans la construction des savoirs sur une difficulté orthographique majeure du français ». Langue française 151 (2006) : 74-93.

Brissaud, Catherine, et Jean-Michel Sandon. «L'acquisition des formes verbales en /E/ à l'école élémentaire et au collège, entre phonographie et morphographie ». Langue française 124 (1999) : 40-57.

Catach, Nina, et Fabrice Jejcic. Les listes orthographiques de base du français (LOB). Les mots les plus fréquents et leurs formes flécbies les plus fréquentes. Paris : Nathan-Recherche, 1984.

Chevrot, Jean-Pierre, Catherine Brissaud, et Pascale Lefrançois. « Norme et variations dansl'acquisition de la morphologie verbale en /E/: tendances, conflits de tendances, résolution». Faits de Langues 22 (2003) : 57-66.

Cuq, Jean-Pierre, et Isabelle Gruca. Cours du français langue étrangère et seconde. Grenoble : Presses Universitaires de Grenoble, 2005.

David, Jacques. «L'orthographe verbale du français, entre hypernorme et acquisition ». Le français aujourd'bui HS01 (2011) : 221-231. 
David, Jacques, Odile Guyon, et Catherine Brissaud. "Apprendre à orthographier les verbes: le cas de l'homophonie des finales en /E/ ». Langue française 151 (2006) : 109-126.

Fayol, Michel, et Sébastien Pacton. "L'accord du participe passé : entre compétition de procédures et récupération en mémoire ». Langue française 151 (2006) : 59-73.

Haas, Ghislaine, et Danielle Lorrot. « De la grammaire à la linguistique par une pratique réflexive de l'orthographe ». Repères 14 (1996) : 161-181.

Hafeznia, Mohammad Reza. An Introduction to the Research Method in Humanities. Tehran: SAMT, 2013.

Jaffré, Jean-Pierre. Didactiques de l'orthographe. Paris: Hachette Éducation, 1992.

Jaffré, Jean-Pierre, et Daniel Bessonnat. "Gestion et acquisition de l'accord : erreurs et étiologie ». Faits de langues 8 (1996): 185-192.

. " Accord ou pas d'accord? Les chaines morphologiques». Pratiques 77 (1993) : 25-42.

Katoozian, Katayoon. "Une revue d'articles sur les erreurs orthographiques à dominante phonétique : réflexions pour le FLE ». Linguarum Arena 5 (2014) : 31-45.

"Revue critique de différentes typologies des erreurs d'orthographe française ». Studii si Cercetari Filologice - Seria Limbi Straine Aplicate 13 (2014) : 115-128.

- Analyse des erreurs orthographiques des étudiants iraniens en langue française. Thèse de doctorat en Didactique des langues et des cultures. Université Sorbonne Nouvelle - Paris 3, 2013. . "Comparaison de types d'erreurs orthographiques en FLM et FLE : une revue d'articles ». Actes $d u$ colloque international doctorants et jeunes chercheurs en Sciences du Langage de l'Université de Toulouse 2 - Le Mirail (2013) : 64-69.

Luzzati, Daniel. «L'orthographe dans l'élaboration des niveaux de référence pour le français ». TRANEL (Travaux neuchâtelois de linguistique) 54 (2011) : 7-19.

didactique), 2010.

Le français et son orthographe. Paris: Didier (Coll. Langues \&

Meleuc, Serge, et Nicole Fauchart. Didactique de la conjugaison. Le verbe "autrement». Paris : Bertrand-Lacoste / Toulouse : CRDP MidiPyrénées (Coll. «DIDACTIQUES »), 1999.

Mialaret, Gaston. Les méthodes de recherche en science de l'éducation. Paris: Presses Universitaires de France, 2004.

New, Boris, Christophe Pallier, Ludovic Ferrand, et Rafael Matos. «Une base de données du français contemporain sur internet: LEXIQUE ». L'Année Psychologique 101 (2001) : 447-462. 
Suleiman, Shehadeh, et Nouman Malkawi. «L'Orthographe, le Grand Absent des MéthodesF.L.E. ». Cross-Cultural Communication 8-6 (2012) : 125-135. Web.

Surcouf, Christian. «L'enseignement et l'apprentissage de la conjugaison en FLE : comment réduire les difficultés engendrées par l'orthographe? ». TRANEL (Travaux neuchatelois de linguistique) 54 (2011) : 93-112. 